\title{
Topical nano-delivery of 5-fluorouracil: Preparation and characterization of water-in-oil nanoemulsion
}

\author{
Rajinikanth Siddalingam* and Kumarappan Chidambaram \\ Department of Pharmaceutical Technology, School of Pharmacy, Taylor's University, Subang Jaya, Malaysia, 47500
}

${ }^{*}$ For correspondence: Email: Rajinikanth.PS@taylors.edu.my; Tel: 0060-0356295000 ext 5422; Fax: 0060-0356295001

Received: 7 May 2016

Revised accepted: 9 October 2016

\begin{abstract}
Purpose: To prepare and characterize a water-in-oil nanoemulsion of 5-fluorouracil (5FU) for enhanced skin penetration.

Methods: Nanoemulsions of 5FU were prepared using Capyrol (propylene glycol monocaprylate). Transcutol (highly purified diethylene glycol monoethyl ether) and polyethylene glycol (PEG) 400 as oil, surfactant and co-surfactant, respectively. The optimized formulations were subjected to heating cooling cycling, centrifugation and freeze - thaw cycling to assess their stability. Particle size distribution and zeta potential of the nanoemulsions were evaluated. Furthermore, in vitro and in vivo skin permeation studies were carried out on the formulations in a rat model. Skin irritation studies were also performed on rats to assess the irritation potential of the formulations. The $1 \% \mathrm{w} / \mathrm{v}$ of Carbopol $934 \mathrm{gel}$ loaded with $1 \% 5 F U$ was used as control (FU gel).

Results: The results showed that the mean droplet size of the nanoemulsions was $\sim 100 \mathrm{~nm}$ with a zeta potential of \pm 15 . Significant increase in permeability was also observed for the nanoemulsion formulations compared with control. The steady-state flux (Jss), enhancement ratio and permeability coefficient $(\mathrm{Kp})$ for optimized nanoemulsion formulation were significantly $(p<0.05)$ higher than those of the conventional gel (control). Both in vitro and in vivo skin retention results indicate higher drug release from the nanoemulsion $\left(292.45 \mu \mathrm{g} / \mathrm{cm}^{2}\right)$ than for control $\left(121.42 \mu \mathrm{g} / \mathrm{cm}^{2}\right)$. Mean irritation index for the nanoemulsion was significantly lower than for control.

Conclusion: The results suggest that a water-in-oil nanoemulsion is safe and can potentially be used to promote skin penetration of 5FU following topical application on the skin for the treatment of some skin diseases.
\end{abstract}

Keywords: Nanoemulsion, Controlled release, 5-Fluorouracil, Skin penetration, Skin irritation

Tropical Journal of Pharmaceutical Research is indexed by Science Citation Index (SciSearch), Scopus, International Pharmaceutical Abstract, Chemical Abstracts, Embase, Index Copernicus, EBSCO, African Index Medicus, JournalSeek, Journal Citation Reports/Science Edition, Directory of Open Access Journals (DOAJ), African Journal Online, Bioline International, Open-J-Gate and Pharmacy Abstracts

\section{INTRODUCTION}

5 -Fluorouracil (5FU) is an antineoplastic agent used for the treatment of skin diseases such as psoriasis, cutaneous premalignant, malignant lesions and basal-cell carcinoma [1]. However, in each instances less than optimal delivery of 5-FU using conventional preparations has necessitated the use of more invasive measures to obtain therapeutic outcomes. The ideal drug delivery for most skin diseases is for the drug to be able to penetrate deeper skin layers. As such the drug delivery strategy should be made to localize high drug concentrations inside the epidermis and dermis layers for effective therapy [2]. There has been an increased interest during recent years in the use of topical vehicle systems using permeation enhancers [3], but use of these chemical enhancers may be harmful, especially in chronic application. Therefore, topical vehicle 
systems could be made safer without chemical enhancers to facilitate drug permeation through the skin. One of the most promising formulation for enhancement of skin permeation of drugs is nanoemulsion [4].

Nanoemulsions are thermodynamically stable transparent (translucent) dispersions of oil and water stabilized by an interfacial film made by surfactant and co-surfactant molecules having a droplet size of less than $100 \mathrm{~nm}$. The antiproliferative 5-FU has been extensively used as a topical chemotherapy for most skin diseases like cutaneous premalignant, malignant lesions and skin cancer (Basal-cell carcinoma [5]. Therefore, an improved 5FU nanoemulsion formulation with a high degree of permeation could be useful in the treatment various skin diseases. The present study describes the potential of nanoemulsion systems for topical delivery of $5 \mathrm{FU}$ that achieve the goal of increased skin permeation without the use of additional permeation enhancers.

\section{EXPERIMENTAL}

\section{Materials}

5-Fluorouracil was purchased from Spectrum Chemicals, Malaysia. Capryol 90, Labrasol, Transcutol HP, Capyrol PGMC, Lauroglycol- 90, Plurol oleique CC497, Maisine 35-1, Solutol were gifted by Gattefosse, France. Span 80 Tween 60 and Tween 80 were purchased from R\&M Marketing, United States of America (USA). PEG 300 and PEG 400 were acquired from Aldrich, Germany. Ethanol and Span 80 were obtained from Fisher Chemicals and Fluka, USA respectively. Other chemicals used were of analytical reagent grade. All chemicals were used as received.

\section{Solubility studies}

The solubility of $5 \mathrm{FU}$ in various oils was determined by adding excess amount of the drug in $2 \mathrm{~mL}$ of selected oils, surfactants and cosurfactants in $5 \mathrm{~mL}$ vials, which were mixed thoroughly using a vortex mixer [6]. The vials placed in water bath - shaker for $24 \mathrm{~h}$ and the temperature was kept at $25{ }^{\circ} \mathrm{C}$. After that, the mixtures were spun using a centrifuge (Bench Top Refrigerated, Eppendorf) at $3000 \mathrm{rpm}$ for 15 min. The supernatants were sampled and quantified using a HPLC method. A Shimadzu HPLC system (Shimadzu, Japan) with two LC10AT VP pumps with UV detector set at $260 \mathrm{~nm}$ and a RP C-18 column (150 mm x $4.6 \mathrm{~mm}$ I.D.; particle size 5 was used to quantify the $5 \mathrm{FU}$ content in solution.

\section{Construction of pseudoternary phase diagrams}

The ternary the pseudo-ternary phase diagram has been constructed based solubility of drug in each solvent (oil, surfactant and co-surfactant) [7]. The surfactant and co-surfactant were mixed in different ratios $(1: 1,2: 1$, and $3: 1)$. Phase diagrams were constructed for each ratio of mixture of surfactant - co-surfactant $\left(\mathrm{S}_{\mathrm{mix}}\right)$ and oils. For every phase diagram, the proportion of oil and $S_{\text {mix }}$ varies in different volume ratio from $0: 10$ to $10: 0$. The percentage of the aqueous phase was determined using an aqueous titration method [8]. Slow titration of distilled water was added ( $5 \%$ addition at a time) into the $\mathrm{S}_{\text {mix }}$ - oil mixture and the observation of the transition from clear to turbid point was recorded. A calculation was made to determine the percentage of water, oil and $S_{\text {mix }}$ present at the point of turbidity. With the obtained individual percentage, a pseudoternary phase diagram was constructed with the clear-solution region marked and defined as the best emulsification region.

\section{Preparation of nanoemulsion}

From each phase diagram constructed, different points were selected from nanoemulsion region for incorporation surfactant, co-surfactant and oil. $1 \%(\mathrm{w} / \mathrm{w})$ of $\mathrm{FU}$ was dissolved in aqueous phase (distilled water) of all selected nanoemulsion formulation. Then, a selected quantity of $S_{\text {mix }}$ (mixture of surfactant and co-surfactant) was added into the aqueous solution of the drug. The mixtures were stirred for $2 \mathrm{~min}$ and sonicated until the drug dissolved completely. The oil phase (Capyrol PGMC) was added slowly with continuous stirring. The resulting mixtures were then passed through a high pressure homogenizer (Avestin Emulsifex C3) for 5 cycles.

\section{Thermodynamic stability testing}

The formulations were subjected to heatingcooling, centrifugation and freeze-thaw stability testing programme, where the physical appearances of the formulations were observed at each testing period. In the heating-cooling programme, all six formulations were heated at $45^{\circ} \mathrm{C}$ and then cooled at $4{ }^{\circ} \mathrm{C}$, with a duration of $24 \mathrm{~h}$ at each temperature, for 2 cycles. Then, formulations which passed the heating-cooling cycles were subjected to centrifugation at 3500 rpm for 15 min. Finally, only formulations which passed the previous two steps were stored at alternating temperature of -21 and $25{ }^{\circ} \mathrm{C}$, with a 
duration of $24 \mathrm{~h}$ at each temperature, for 2 cycles.

\section{In vitro skin permeation studies}

Skin of 8-9 weeks old Swiss albino mice weighing 25 to $30 \mathrm{~g}$ was mounted in a Franz diffusion cell. The skin was washed with normal saline then dried between two filter papers. The skin was then carefully checked through a magnifying glass to ensure that samples were free from any surface irregularities such as tiny holes or cervices in the portion. $2 \mathrm{~cm}^{2}$ of the excised skin samples were clamped between the donor and receptor chamber of a Franz diffusion cell with the stratum corneum facing the donor chamber. Then, $2 \mathrm{ml}$ of prepared nanoemulsion formulations were administered into the donor chamber. The receptor chamber was filled with $15 \mathrm{ml}$ of phosphate buffer solution (PBS) $\mathrm{pH}$ 7.4. The receptor medium was maintained at $37 \pm 0.5$ ${ }^{\circ} \mathrm{C}$ and stirred at $500 \mathrm{rpm}$ throughout the experiment. $0.5 \mathrm{ml}$ of sample was collected from the receptor medium of each cell every hour for 8 hours and then immediately replaced by the same amount of preheated PBS. The collected samples were filtered through a $0.45 \mu \mathrm{m}$ pore size cellulose membrane filter and analyzed using a HPLC method as described above [9]. All animal protocols were approved by the Animal Ethics Committee of International Medical University, Malaysia (approval ref. no IAEC/BP/2012/0087). The animal experiment was performed in accordance with Directive 2010/63/EU on the handling of animals used for scientific purposes [10].

\section{Permeation data analysis}

The cumulative amount of drug permeated through the skin $\left(\mu \mathrm{g} / \mathrm{cm}^{2}\right)$ was plotted against time in hours for each formulation. The drug flux (permeation rate) at steady-state $\left(\mathrm{J}_{\mathrm{ss}}\right)$ and permeability coefficient (KP) were calculated from the slope of the linear portion of the graph using Eq 1 [11].

$\mathrm{KP}=\mathrm{J}_{\mathrm{SS}} / \mathrm{C}_{0}$

where, $\mathrm{J}_{\mathrm{ss}}$ is the drug flux at steady state and $\mathrm{C}_{0}$ is the initial drug concentration in the donor cell. Enhancement ratio (Er) was calculated by dividing the $J_{s s}$ of respective formulation with $J_{s s}$ of control formulation as in Eq 2.

$\mathrm{Er}=\mathrm{J}_{\mathrm{ss}} \mathrm{F} / \mathrm{J}_{\mathrm{ss}} \mathrm{C}$

where, $J_{s s} F$ is the $J_{s s}$ of formulation and $J_{s s} C$ is the $\mathrm{J}_{\mathrm{ss}}$ of control.
The significance of results was checked statistically at $p<0.05$ applying a one-way ANOVA test. Post hoc multiple comparisons were carried out using the least square difference test [12].

\section{In vivo skin deposition studies}

8-9 weeks old male Swiss albino mice weighing 20 to $25 \mathrm{~g}$ were used for this study. The animals were divided into 5 groups, with each group consisting of 5 animals. Prepared nanoemulsion FU2, placebo formulation (control), $5 \%$ SLS and plain carbopol gel containing $1 \% 5 \mathrm{FU}$ (plain gel) were applied to the dorsal surface $\left(3 \mathrm{~cm}^{2}\right)$ of each group of mice after removal of its hair by clipping. The mice were then put to death by cervical dislocation at the end of $1,2,4$ and 8 hours after dorsal application and the administered skins were stripped and adhering fats and debris were removed carefully. The skin was thoroughly washed with methanol and then dried. The drug content in the dermis skin layer was determined as described in the in vitro skin deposition study and drug content was analyzed by the HPLC method.

\section{Skin irritation studies}

Wistar albino rats (16 weeks) weighing 200 - 250 $g$ were used in this part of the study [12]. The animals were kept under standard laboratory conditions, with standard temperature of $25^{\circ} \mathrm{C} \pm$ $1{ }^{\circ} \mathrm{C}$ and relative humidity of $60 \%$. They had free access to a standard laboratory diet with water ad libitum. Prior to the application of formulations onto the animals, the hairs on the dorsal surface of the rats were removed by clipping. Five rats were assigned to each treatment groups: Group 1 received placebo formulation (negative control), Group 2 received sodium lauryl sulphate (SLS) solution (5\% w/v, positive control), Group 3 received prepared FU2 nanoemulsion formulation and Group 4 received carbopol gel containing $1 \%$ 5FU. $200 \mu \mathrm{l}$ of these prepared samples were applied onto the dorsal surface of the rats daily for six days using the reported method [13]. The rats were observed and erythema scores ranging from 0 to 4 were allocated depending upon the degree of erythema as follows, 0: No erythema 1: Slight erythema (barely perceptible light pink). 2: Moderate erythema (dark pink). 3: Moderate to severe erythema (light red). 4: Severe erythema (extreme redness).

\section{Statistical analysis}

Data are presented as mean \pm standard (SD). The difference in results between batches 
containing various portion of excipients was statistically analyzed using Student's t-test with the aid of SPSS 16.0 statistical software. Differences were considered statistically significant at $p<0.05$.

\section{RESULTS}

\section{Solubility}

In order to select the suitable solvents for the preparation of nanoemulsions, the solubility of 5FU various oil, co-surfactants and surfactants were determined and the results are shown in Figure1. The drugs that 5-FU has shown good solubility in are Trancutol HP, Capyrol PGMC and PEG 400 with a solubility of 94.62, 78.94 and $93.12 \mathrm{mg} / \mathrm{mL}$ respectively.

\section{Construction of pseudoternary phase diagrams}

A phase diagram was constructed on the basis of the solubility of drug, oil, surfactants, cosurfactants and aqueous phase. The aqueous titration was performed with addition of water (5 $\%$ increment of up to $95 \%$ [14]. As shown in Figure 2, the area of nanoemulsion region in the phase diagram appeared to have decreased minimally when the $S_{\text {mix }}$ ratio increases. The $S_{\text {mix }}$ $1: 1$ shows a larger nanoemulsion region than that of $3: 1$.

It was also noticeable that the $S_{\text {mix }} 1: 1$ ratio took up a greater amount of water and still remained as a clear mixture during aqueous titration. In Figure 2, the region $\mathrm{A}$ forms a clear emulsion while $B$ \& $C$ formed more turbid and milky emulsions.

\section{Preparation of nanoemulsion}

The nanoemulsifying region incorporating $1 \%$ W/V of $5 F U$ in $S_{\text {mix }} 1: 1$ appeared to be larger $(p<$ $0.05)$ than that of the other $S_{\text {mix }}$ formulation ratios (1:2 and 1:3) in the presence of drug (Figure 2(a)). Six points were randomly picked from the self-nanoemulsifying region from $S_{\text {mix }} 1: 1$ and each formulations composition are shown in Table 1.

\section{Droplet size}

The mean droplet size of 5 -FU nanoemulsions was in the range of $80.24-112.87 \mathrm{~nm}$. The droplet size of nanoemulsion was increased with the increase in concentration of oil (Capyrol PGMC) as shown in Table 2.

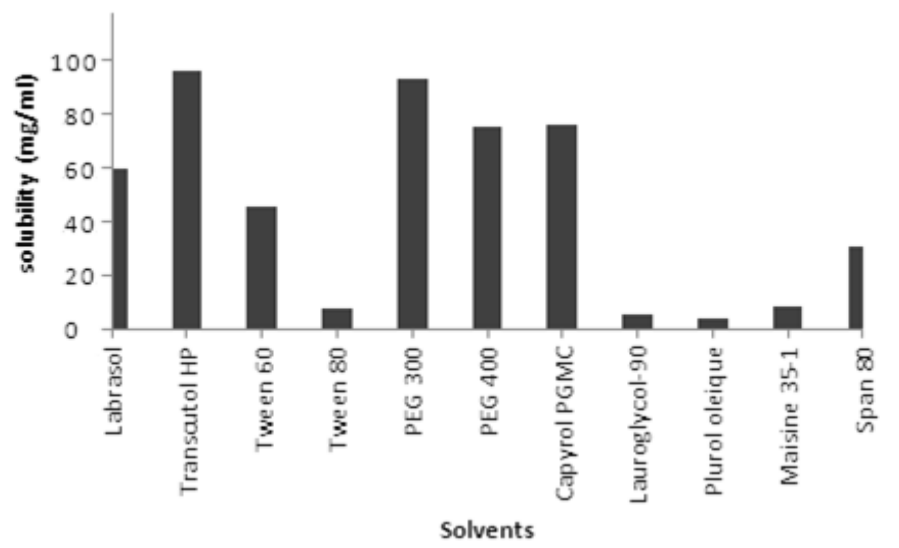

Figure 1: Phase solubility of 5-FU in various oils, surfactant and co-surfactants

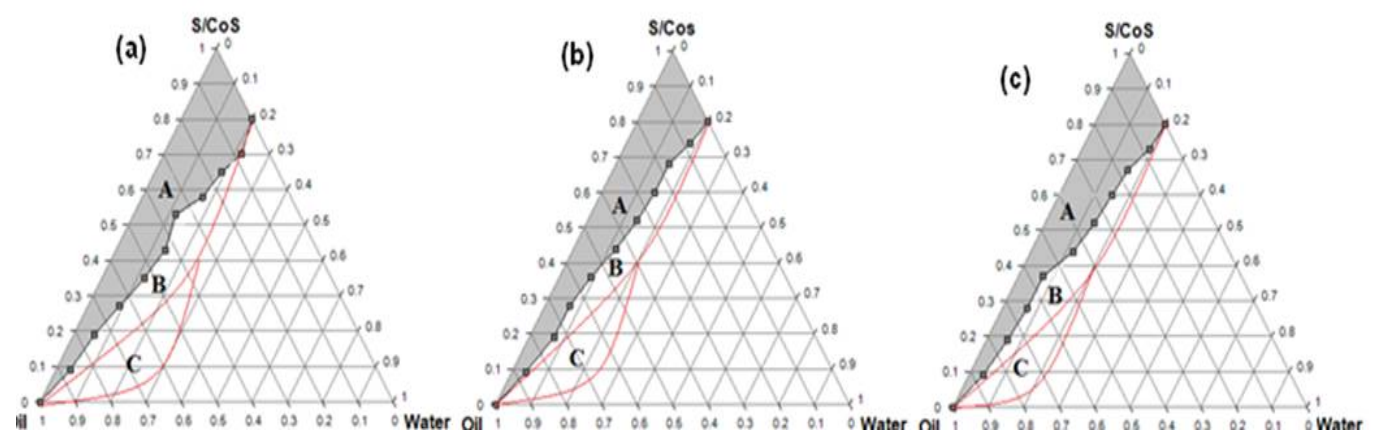

Figure 2: Pseudoternary phase diagrams of Capyrol PGMC, Transcutol and PEG 400 with 100 mg of 5FU. a) $S_{\text {mix }} 1: 1$; b) $S_{\text {mix }} 2: 1$ c) $S_{\text {mix }} 3: 1$ 
Table 1: Composition of selected water-in-oil nanoemulsion of 5-fluorouracil

\begin{tabular}{lcccc}
\hline Batch code & \multicolumn{3}{c}{ Components $\mathbf{( \% w / w )}$} & S $_{\text {mix }}$ ratio \\
\cline { 2 - 5 } & Oil & $\mathbf{S}_{\text {mix }}$ & Water $^{*}$ & \\
\hline FU1 & 50.0 & 40.0 & 10.0 & $1: 1$ \\
FU2 & 55.0 & 40.0 & 10.0 & $1: 1$ \\
FU3 & 60.0 & 30.0 & 10.0 & $1: 1$ \\
FU4 & 50.0 & 40.0 & 10.0 & $1: 1$ \\
FU5 & 50.0 & 35.0 & 15.0 & $1: 1$ \\
FU6 & 50.0 & 30.0 & 20.0 & $1: 1$ \\
\hline${ }^{*}$ Aqueous phase (water) containing $1 \%$ w/v of $5 \mathrm{FU}$ &
\end{tabular}

The formulation containing $60 \%$ (FU3) oil has the highest particle size (112.87 \pm 1.78$)$ and formulation containing $20 \%$ water and $50 \%$ oil has the lowest particle size $(80.24 \pm 2.14) \mathrm{nm})$ as compared to other formulations. The results from Table 2 show that all the formulations had droplets in the nanometer range with low values of polydispersity index.

\section{Thermodynamic stability}

The results of stability testing for the nanoemulsion are shown in Table 3. All of the optimized formulations passed the thermodynamic stability testing (freeze thaw cycle) as none of them exhibited any sign of phase separation or drug precipitation at the end of the cycles.

\section{In vitro skin permeation}

The in vitro release profiles of $5 \mathrm{FU}$ from its various nanoemulsion formulations containing 1 $\% \mathrm{w} / \mathrm{v}$ of $5 \mathrm{FU}$ (FU1, FU2, FU3, FU4) and plain carbopol gel containing $1 \%$ of $5 \mathrm{FU}$ are presented in Figure 3. The in vitro skin permeation was found to be highest for formulation FU1 and lowest for FU3 (Figure 3). The skin permeation profile of FU1 was significantly $(p<0.05)$ higher as compared to FU3. In vitro skin permeation parameters of prepared nanoemulsion formulations and control are shown in Table 4.

It was observed that the permeation parameters cumulative amounts of drug permeated, steadystate flux values, enhancement ratios and permeability coefficients in all nanoemulsion based formulation (FU1, FU2, FU3 and FU4) were found to be significantly $(p<0.05)$ higher than that of FU loaded conventional gel at the end of the $8 \mathrm{~h}$ permeation study as shown in Table 4. The amount of drug permeated from its nanoemulsion formulation through the skin can be arranged in the following descending order: FU1 $>$ FU3 $>$ FU2 $>$ FU4, where the amounts of the drug penetrated after the $8 \mathrm{~h}$ of study were 196.36, $156.25,136.25$ and $112.21 \mu \mathrm{g} / \mathrm{cm}^{2}$, respectively. In contrast, in vitro skin permeation of conventional gel containing $1 \% 5 \mathrm{FU}$ was observed to be significantly $(p<0.05)$ lower $\left(60.12 \mu \mathrm{g} / \mathrm{cm}^{2}\right)$ at the end of $8 \mathrm{~h}$.

Table 2: Mean droplet size, polydispersity index and zeta potential $(\mathrm{mV})$ of each formulation used in the study

\begin{tabular}{lccc}
\hline Formulation & $\begin{array}{c}\text { Mean droplet size } \\
(\mathbf{n m})\end{array}$ & Polydispersity index & Zeta potential $(\mathbf{m V})$ \\
\hline FU1 & $82.28 \pm 1.98$ & $0.328 \pm 0.040$ & $-11.87 \pm 0.124$ \\
FU2 & $93.54 \pm 2.17$ & $0.228 \pm 0.040$ & $-16.21 \pm 0.141$ \\
FU3 & $112.87 \pm 1.78$ & $0.310 \pm 0.021$ & $-15.25 \pm 0.142$ \\
FU4 & $98.45 \pm 1.87$ & $0.211 \pm 0.032$ & $-16.30 \pm 0.378$ \\
FU5 & $97.51 \pm 1.28$ & $0.397 \pm 0.047$ & $-10.71 \pm 0.247$ \\
FU6 & $80.24 \pm 2.14$ & $0.258 \pm 0.041$ & -13.930 .387 \\
\hline
\end{tabular}

Table 3: Mean droplet size, polydispersity index (PDI) and zeta potential (mV) of the optimized nanoemulsions after exposure to a freeze thaw cycle

\begin{tabular}{lccc}
\hline Sample & Droplet size $(\mathbf{n m})$ & PDI & Zeta potential \\
\hline FU1 & $81.24 \pm 2.27$ & $0.218 \pm 0.030$ & $-13.45 \pm 0.244$ \\
FU2 & $93.27 \pm 2.19$ & $0.317 \pm 0.031$ & $-14.21 \pm 0.141$ \\
FU3 & $109.27 \pm 2.14$ & $0.288 \pm 0.028$ & $-15.25 \pm 0.134$ \\
FU4 & $96.78 \pm 2.78$ & $0.218 \pm 0.031$ & $-16.30 \pm 0.289$ \\
FU5 & $97.89 \pm 2.47$ & $0.309 \pm 0.044$ & $-10.71 \pm 0.315$ \\
FU6 & $84.47 \pm 2.12$ & $0.271 \pm 0.056$ & $-14.58 \pm 0.282$ \\
\hline
\end{tabular}




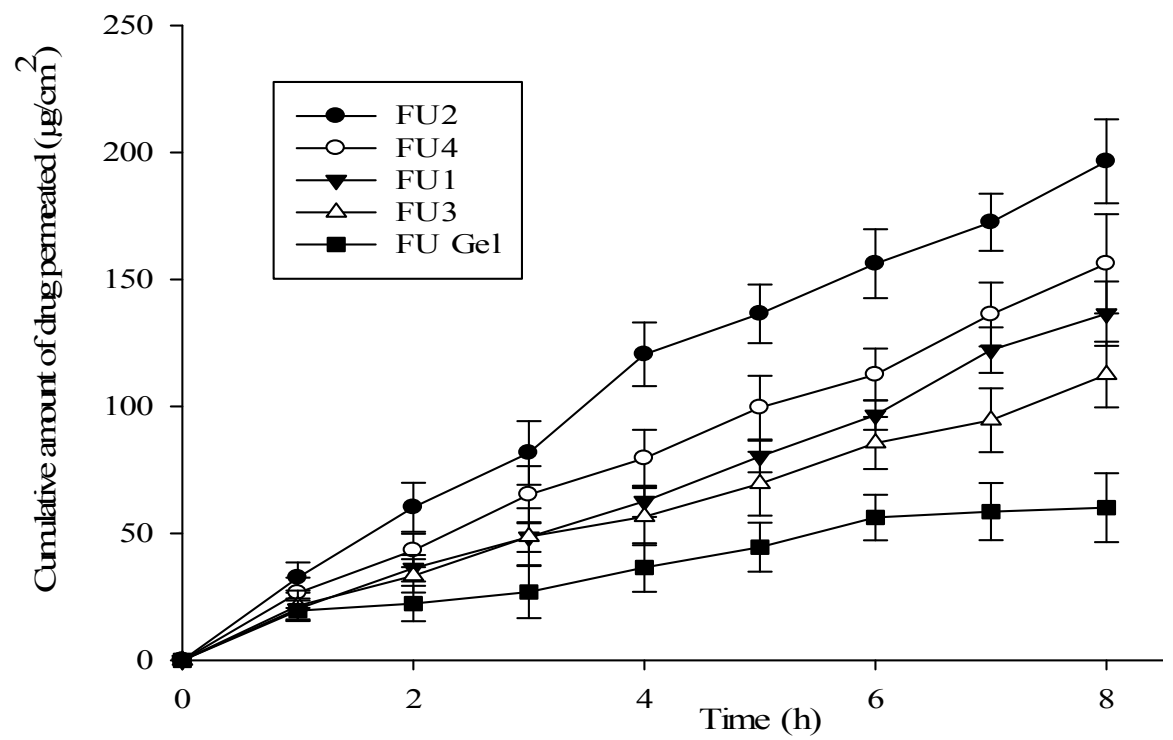

Figure 3: In vitro skin permeation of 5-FU from nanoemulsion through excised mice skin in a Franz diffusion cell at $37 \pm 0.5^{\circ} \mathrm{C}$; mean $\pm \mathrm{SD}(\mathrm{n}=3)$

Table 4: In vitro permeation parameters of $5 \mathrm{FU}$ from nanoemulsions (mean $\pm \mathrm{SD}, \mathrm{n}=3$ )

\begin{tabular}{lcccc}
\hline Formulation & $\begin{array}{c}\text { Steady-state flux } \\
(\mathbf{J s s})\left(\boldsymbol{\mu g} / \mathbf{c m}^{\mathbf{2}} / \mathbf{h}\right)\end{array}$ & $\begin{array}{c}\text { Enhancement } \\
\text { ratio }\end{array}$ & Lag time (hr) & $\begin{array}{c}\text { Permeation } \\
\text { coefficient }(\mathbf{k p}) \\
(\mathbf{c m} / \mathbf{h})\end{array}$ \\
\hline FU1 & $24.21 \pm 2.45$ & $3.28 \pm 0.87$ & $0.89 \pm 0.18$ & $19.52 \pm 1.87$ \\
FU2 & $19.74 \pm 1.98$ & $2.27 \pm 0.48$ & $0.74 \pm 0.17$ & $12.45 \pm 1.24$ \\
FU3 & $13.25 \pm 2.17$ & $1.80 \pm 0.29$ & $0.38 \pm 0.19$ & $11.86 \pm 1.45$ \\
FU4 & $18.78 \pm 1.58$ & $2.54 \pm 0.81$ & $0.75 \pm 0.21$ & $17.45 \pm 1.69$ \\
FU gel & $7.38 \pm 1.57$ & $1.00 \pm 0.19$ & $0.21 \pm 0.02$ & $6.47 \pm 1.27$ \\
\hline
\end{tabular}

\section{In vivo skin deposition}

Based on the in vitro skin permeation results, the nanoemulsion formulation FU1 and the aqueous cream were selected for in vivo skin deposition studies. The amount of drug retained on mice skin for different time intervals $(1 \mathrm{~h}, 2 \mathrm{~h}, 4 \mathrm{~h}$ and 8h) are shown in Figure 4. It was observed that the there was no significant $(p<0.05)$ difference in skin deposition in FU2 at different time intervals and that the maximum amount of deposition $\left(292.45 \mu \mathrm{g} / \mathrm{cm}^{2}\right)$ was reached at the end of $2 \mathrm{~h}$ at which point there was no further increase of drug deposition observed.

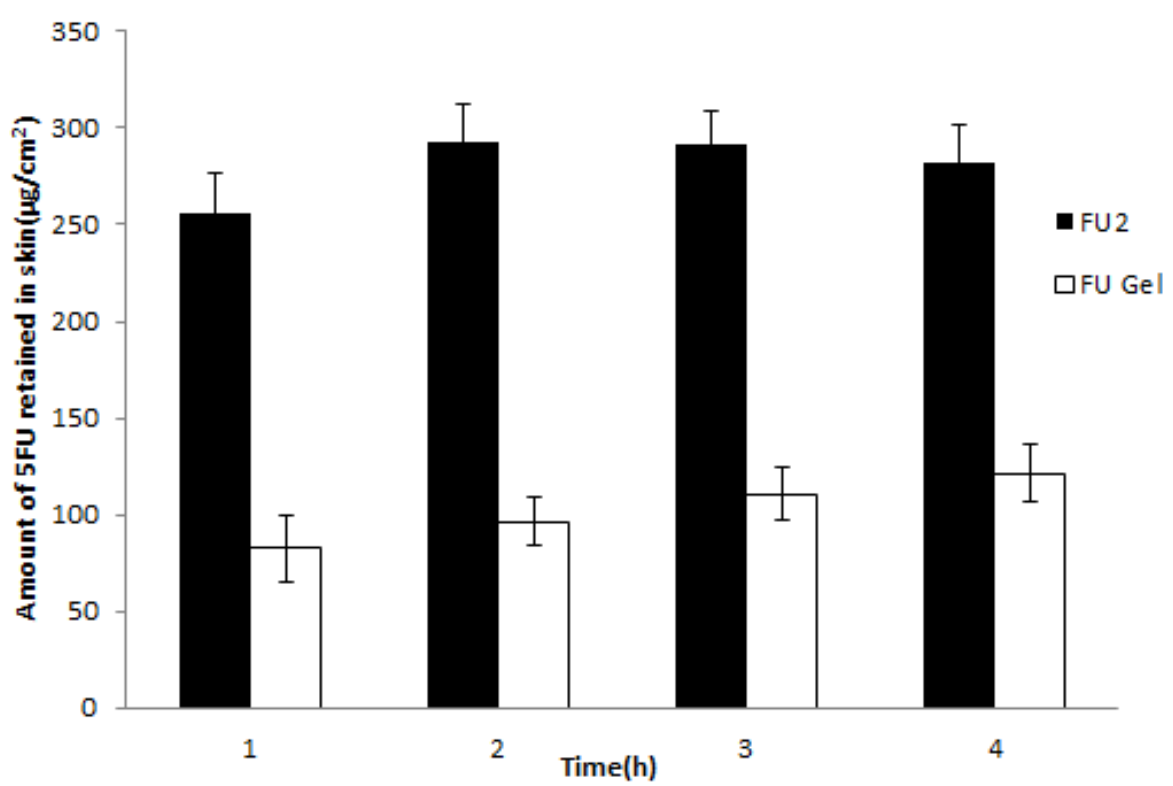

Figure 4: Amount of 5-FU retained in rat skin at the end of 8 hours of in vivo deposition studies; (mean $\pm S D, n=$ 3 
On the other hand, it was noticed that the amount of drug retained in $5 F U$ loaded conventional gel was $121.42 \mu \mathrm{g} / \mathrm{cm}^{2}$ at the end of the $8 \mathrm{~h}$ study and this was attributed to a lower ability of the cream formulation to penetrate the skin layers. Finally, all the nanoemulsion based formulations (FU1, FU2, FU3 and FU4) showed good in vitro penetration, in vitro skin deposition, and in vivo skin deposition in mice skin. Out of all the formulations, FU1 showed the maximum in vitro penetration and in vivo skin deposition as compared to the other formulations (Figure 3 \& 4).

\section{Skin irritation}

The study was conducted to test the safety and skin irritation caused by formulations. The mean erythema scores upon application of optimized nanoemulsion FU1, $5 \%$ W/V SLS solution control (placebo) and carbopol plain gel of $1 \%$ 5FU are shown in Figure 5. The SLS solution (5 $\%$ W/V) was used as standard irritation in this study. Results indicate that erythema caused by SLS were much higher (4.1 level score) compared to FU1 and plain gel containing $1 \%$ $5 F U$.

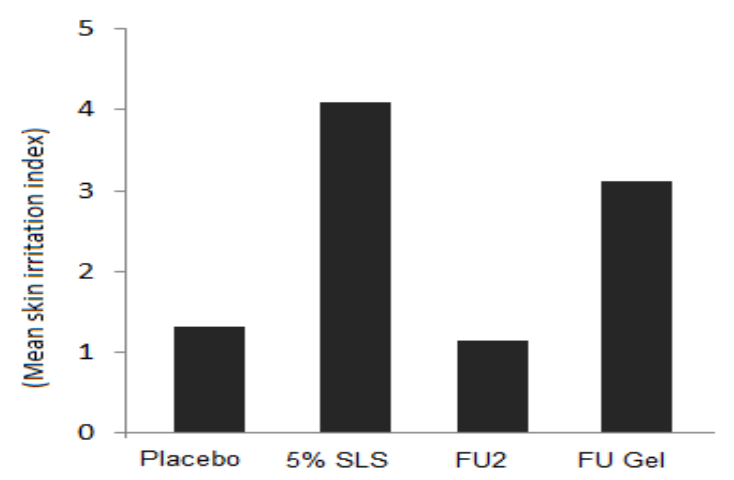

Figure 5: Mean irritation index of various 5-FU nanoemulsion and 5-FU conventaional cream; (mean $\pm \mathrm{SD}, \mathrm{n}=3$ )

\section{DISCUSSION}

The drug has shown the highest solubility in Transcutol HP and it has been chosen as the surfactant for this study. Similarly, PEG 400 and Capyrol PGMC were chosen as the oil and cosurfactant, respectively, for this study. Furthermore, Transcutol HP reported to be very attractive as a penetration enhancer due to its non-toxicity and biocompatibility with the skin [14]. The right blend of oil and surfactants with the right value of hydrophilic lipophilic balance (HLB) leads to the formation of stable nanoemulsion formulations. In this study, HLB value of blend of oils and surfactants were found to be $\sim 5.3$ which is in the range for W/O type of emulsion.

In Figure 2, diagram (a) has shown the largest emulsification area. This could be explained by the fact that a higher number of surfactants can be absorbed at the interface, thus stabilising the formation of nanoemulsions [15]. Hence, the ratio of 1:1 was selected for formulation development and 6 random points within this area was selected to be used for further studies. A pseudoternary phase diagram in the presence of $1 \%$ W/V 5-FU was also plotted with $S_{\text {mix }} 1: 1$ to determine the effect of drug on the phase diagram [16]. $S_{\text {mix }} 1: 1$ ratio showed no difference in the area of nanoemulsion whether with the presence or absence of $1 \% 5 \mathrm{FU}$.

Determining the droplet size is of great importance in nanoemulsion formulations as small globule sized emulsion contributes to a greater interfacial area which can then provide better drug partitioning and absorption. However, there is no consensus in the literature on the exact size range of a nanoemulsion [16]. The polydispersity values of all formulations were very low which indicates uniformity of droplets within the formulation. The Zeta potential of the formulation was also measured in order to determine the interaction between colloidal particles, where large positive or negative values suggesting high inclination of emulsion repulsion and hence, stability [17].

The results from thermodynamic stability studies of the optimized preparation shows good stability against acceleratory stability condition. The average particle size and physical appearance of the formulation after the stability study at extreme conditions was observed and the results indicated that there were no significant changes in particle size or physical appearance (Table 5).

Table 5: Physical appearance of nanoemulsion after exposure to a freeze thaw cycle

\begin{tabular}{lll}
\hline Sample & $\begin{array}{l}\text { Observation } \\
\text { (after first cycle \& } \\
\text { centrifugation) }\end{array}$ & $\begin{array}{l}\text { Observation } \\
\text { (after third cycle } \\
\text { \& centrifugation) }\end{array}$ \\
\hline FU1 & Clear & Clear \\
FU2 & Clear & Clear \\
FU3 & Clear & Clear \\
FU4 & Clear & Clear \\
FU5 & Clear & Cloudy \\
FU6 & Partially crystallized & Partially \\
& & crystallized \\
\hline
\end{tabular}

The significant difference in drug permeation between nanoemulsion formulations FU1 and FU3 was probably due to the mean size droplets [18], which were significantly smaller for FU than 
for FU2 and FU3 and also due to the higher amount of penetration enhancer (Transcutol and Capyrol PGMC) used in the FU1 formulation which may work by interacting with the structural components of the stratum corneum to enhance drug penetration [19].

It was observed that the in vitro skin permeation parameter values of 5FU gel preparation were much lower than that of the nanoemulsion shown in Figure 4. This could be due to the low flux and high viscosity of the formulation which can explain the lesser in ability for the aqueous solution to penetrate skin layers as compared to nanoemulsion formulations as shown in Figure 4. In this study, Transcutol was used as the surfactant in this study and this compound has been reported for its skin penetration-enhancing effect $[18,19]$. Capyrol PGMC was used as the oil in this study and this oil is known for its penetration-enhancing effect by altering the skin texture [20]. Moreover, the characteristics of nanoemulsions such as high drug concentration, the small droplet diameter and the reaction between the surfactants and skin stratum corneum could have been contributed to the permeation-enhancement of the prepared formulations.

From the above in vivo and in vitro permeation data, it was found that all of the prepared nanoemulsions could improve the skin permeation of $5 \mathrm{FU}$ over the conventional gel that has the same drug concentration. This could be, as reported previously, the thermodynamic activity of formulations which can be described as viscosity is important to the permeation into skin. It is known that the viscosity of nanoemulsions is much lower than that of gel, so the mobility of drug in nanoemulsions is more facile. Furthermore, the nanoemulsions may affect the stratum corneum structure and reduce the diffusional barrier by acting as a permeation enhancer [20,21].

In the skin irritation study, the mean irritation score of FU1 was significantly lesser than that of the plain gel. The results clearly indicate that the optimized nanoemulsion formulation was well tolerated by rat skin and produced no irritation to skin. Based on this, it seems that the optimized nanoemulsion formulation may be safe used for topical drug delivery compared to conventional gel or cream formulations of the drug.

\section{CONCLUSION}

The penetration of $5 \mathrm{FU}$ into the skin and its retention within the skin is desirable for the treatment of many skin diseases, including skin cancer. Thus, the developed nanoemulsion formulation has great potential for topical delivery of $5 \mathrm{FU}$ for safe and more effective treatments of various skin diseases including skin cancer. However, further studies, such as extensive pharmacokinetic, histopathological and toxicity studies are required to fully develop these formulations for therapeutic application.

\section{DECLARATIONS}

\section{Acknowledgement}

The authors are grateful to School of Pharmacy, International Medical University, Kuala Lumpur, Malaysia for financial support for this study.

\section{Conflict of Interest}

No conflict of interest associated with this work.

\section{Contribution of Authors}

The authors declare that this work was done by the authors named in this article and all liabilities pertaining to claims relating to the content of this article will be borne by them.

\section{Open Access}

This is an Open Access article distributed under the terms of the Creative Commons Attribution License, which permits unrestricted use, distribution, and reproduction in any medium, provided the original work is properly credited.

\section{REFERENCES}

1. Tsuji T, Sugai T. Topically Administered Fluorouracil in Vitiligo. Arch Dermatol 1983; 119(9): 722-727.

2. Kong $M$, Chen XG, Kwon DK, Park HJ. Investigations on skin permeation of hyaluronic acid based nanoemulsions as transdermal carrier. Carbohydrate Polymers 2011; 86(2): 837-843

3. Robertsand WJ, Sloan KB. Topical delivery of 5fluorouracil (5-FU) by 3-alkylcarbonyloxymethyl-5-FU prodrugs. J Pharm Sci 2003; 92: 1028-36.

4. Khandavilli S, Panchagnula R. Nanoemulsions as Versatile Formulations for Paclitaxel Delivery: Peroral and Dermal Delivery Studies in Rats. J. Investig Dermatol 2007; 127: 154-162.

5. Kakumanu SS, Tagne JB, Wilson TA, Nicolosi RJ. A nanoemulsion formulation of dacarbazine reduces tumor size in xenograft mouse epidermoid carcinoma model compared to dacarbazine suspension. Nanomed: Nanotech Bio Med 2011; 7(3): 277-283.

Trop J Pharm Res, November 2016; 15(11): 2318 
6. Rajinikanth PS, Woei Keat N, Garg S. Development and In-Vitro characterization of Self-nanoemulsifying Drug Delivery Systems of Valsartan Intl J Drug Delivery 2012; 4(2): 30-41.

7. Sheikh SN, Faiyaz S, Sushma T, Javed A, Sanjula B, Alka A, Roop KK, Ali M. Formulation Development and optimization Using Nanoemulsion Technique: A Technical Note. AAPS Pharm SciTech 2007; 8(2): Article 28.

8. Reeta RG, Swantrant KJ, Varshneya M. AOT water-in-oil microemulsions as a penetration enhancer in transdermal drug delivery of 5-fluorouracil. Colloids and Surfaces B: Biointerfaces, 2005; 41: 25-32.

9. Vipasha $D$, Shiwang S, Subheet J, Sandeep A, Arvind S. Formulation characterization and evaluation of new topical 5-FU by drug entrapment in oleic acid vesicles. American J Pharm Res, 2011; 1(2): 1-15.

10. European Commission [homepage on the internet]. Directive 2010/63/EU on the protection of animals used for scientific purposes [cited 2013 Jan 16]. Available from: http://ec.europa.eu/environment/chemicals/lab_ani mals/legislation_en.htm.

11. Das MK, Bhattacharya A, Ghosal SK. Effect of different terpene-containing essential oils on percutaneous absorption of trazodone hydrochloride through mouse epidermis. Drug Delivery. 2006; 3: 425-431.

12. Shakeel F, Baboota S, Ahuja A, Ali J, Aqil M, Shafiq S. Nanoemulsions as vehicles for transdermal delivery of aceclofenac. AAPS PharmSciTech 2007; 8(4):104109.

13. Aliaa N, EIMeshad I, Ibrahim MT. Transdermal Delivery of an Anti-Cancer Drug via W/O Emulsions Based on Alkyl Polyglycosides and Lecithin: Design, Characterization, and In Vivo Evaluation of the Possible Irritation Potential in Rats. AAPS PharmSciTech 2011; 12(1): 1-9.
14. Hong J, Kim J, Song Y, Park J, Kim CK. A new selfemulsifying formulation of itraconazole with improved dissolution and oral absorption. J Control Rel 2006; 110: 332-338.

15. Abhijit $A D$, Neha $D$, Rahul $D$, Mangal $N$. Selfnanoemulsifying drug delivery systems: formulation insights, applications and advances. Nanomedicine 2010; 5(10): 1595-1616.

16. Mohammad C, Jagdish C, Amit KD. Transdermal permeation of tetracaine hydrochloride by lecithin microemulsion. Colloids and Surfaces B: Biointerfaces 2006; 48(1): 58-66.

17. Yin YM, Cui FD, Mu CF, Choi MK, Kim JS, Chung SJ, Shim C.K, Kim DD. Docetaxel microemulsion for enhanced oral bioavailability: preparation and in vitro and in vivo evaluation, J Controlled Rel 2009; 140(2): 86-94.

18. Weiwei Z, Chenyu G, Aihua Y, Yan G, Fengliang C, Guang Z. Microemulsion-based hydrogel formulation of penciclovir for topical delivery. Intl J Pharm 2009; 378(1): 158-162.

19. Guo C, Yang C, Li Q, Tan Q, Liu W, Zhai G. Development of a Quercetin-loaded nanostructured lipid carrier formulation for topical delivery. Intl J Pharm 2012; 430: 392-398.

20. Müller-Goymann CC. Physicochemical characterization of colloidal drug delivery systems such as reverse micelles, vesicles, liquid crystals and nanoparticles for topical administration. Eur J Pharm Biopharm 2004; 58(2): 343-356.

21. Yilmaz E, Borchert $H$. Design of a phytosphingosinecontaining, positively-charged nanoemulsion as a colloidal carrier system for dermal application of ceramides. Eur J Pharm Biopharm 2005; 60(1): 91-98. 